Published in final edited form as:

J Chromatogr B Analyt Technol Biomed Life Sci. 2010 January 1; 878(1): 92-96. doi:10.1016/j.jchromb. 2009.11.015.

\title{
Quantifying the HIV-1 integrase inhibitor raltegravir in female genital tract secretions using high-performance liquid chromatography with ultraviolet detection*
}

\author{
Jasmine A. Talameh ${ }^{a}{ }^{*}$, Naser L. Rezk ${ }^{a, b}$, and Angela D.M. Kashubaa,b \\ aDivision of Pharmacotherapy and Experimental Therapeutics, University of North Carolina at \\ Chapel Hill, Eshelman School of Pharmacy, Campus Box \#7569, Chapel Hill, NC 27599, United \\ States \\ ${ }^{b}$ Clinical Pharmacology and Analytical Chemistry Core, Center for AIDS Research, University of \\ North Carolina at Chapel Hill, 3319 Kerr Hall, CB\#7569, Chapel Hill, NC 27599, United States
}

\begin{abstract}
Understanding the pharmacokinetics of drugs in peripheral body compartments, such as the genital tract, is particularly important in the infectious diseases arena. However, extracting drugs from small volumes of viscous, proteinacious substances like cervicovaginal fluid is particularly challenging. The goal of this study was to develop a method to quantify raltegravir, an HIV-1 integrase inhibitor, in the female genital tract. The method included sample preparation with perchloric acid followed by solid-phase extraction, separation with reverse-phase high-performance liquid chromatography, and detection with an ultraviolet wavelength of $218 \mathrm{~nm}$. The method was linear from 0.05 to 10.0 $\mathrm{mg} / \mathrm{L}$, with minimal endogenous interference. The method was accurate (1.2-11.0\% deviation) and precise (1.1-12.6\% CV) for both within and between-day analyses. The ability to detect raltegravir in the female genital tract is essential for future investigations of raltegravir as an agent for prevention of HIV acquisition, and this method will be used for clinical studies further evaluating pharmacokinetic-pharmacodynamic relationships in this body compartment.
\end{abstract}

\section{Keywords}

Raltegravir; Integrase inhibitor; Female genital tract; Cervicovaginal fluid; Solid-phase extraction; High-performance liquid chromatography

\section{Introduction}

The majority of HIV infections result from heterosexual transmission [1]. Therefore, to abate the HIV epidemic, interventions to prevent the sexual transmission of HIV are needed. Preand post-exposure prophylaxis of HIV transmission using oral antiretroviral (ARV) drugs is a potential approach to prevention [2]. Raltegravir, a novel HIV integrase inhibitor, holds promise as an HIV prophylactic agent due to its mechanism of action and its effectiveness in HIV treatment [3]. However, the effectiveness of raltegravir for this purpose may rely on its

\footnotetext{
*This publication was made possible with help from the UNC Center for AIDS Research (CFAR), an NIH funded program \#P30 AI50410. The following reagent was obtained through the AIDS Research and Reference Reagent Program, Division of AIDS, NIAID, NIH: RALTEGRAVIR from Merck \& Company, Inc.

(c) 2009 Elsevier B.V. All rights reserved.

*Corresponding author. Tel.: +1 919966 5904; fax: +1 919966 5863. jtalameh@email.unc.edu (J.A. Talameh).
} 
ability to accumulate in female genital tract (FGT) secretions. Therefore, in order to be considered for further study in pre- and post-exposure prophylaxis against HIV infection, the pharmacokinetics of raltegravir in female genital tract secretions must be understood.

Currently, two approaches have been published to collect FGT fluid for detection of ARVs: pelvic lavage [4] and direct aspiration [5]. Although a much simpler analytical approach can be used with lavage fluid, the extent to which secretions are diluted can only be estimated, and hence this collection strategy is only semi-quantitative at best. Since lavage can result in potentially misleading results in pharmacokinetic and pharmacodynamic studies, in which accurate measures of drug concentration are crucial [4,5], the goal of this study was to develop an analytical method to measure raltegravir in direct aspirates of CVF using a previously validated solid-phase extraction (SPE) and high-performance liquid chromatography (HPLC) method for measuring raltegravir in plasma [6].

\section{Materials and methods}

\subsection{Chemicals and solvents}

Raltegravir monopotassium salt was obtained from Merck Research Laboratories (Rahway, NJ, USA). Diazepam, used as the internal standard, was purchased from Sigma Chemical Company (St. Louis, MO, USA). HPLC-grade solvents (water, methanol, and acetonitrile), ammonium acetate, trifluoroacetic acid (TFA), perchloric acid (PCA), glacial acetic acid, and tetrabutylammonium phosphate (TBAP) were purchased from Fisher Scientific (Norcross, GA, USA). Human free drug CVF was pooled from healthy donors at the University of North Carolina at Chapel Hill. Purified compressed nitrogen gas was obtained from National Welders Supply (Charlotte, NC, USA).

\subsection{Equipment}

A high-performance liquid chromatography (HPLC) system consisting of an Agilent Technologies (Wilmington, DE, USA) Model HP1100 binary pump, an HP1100 degasser, and HP1100 auto sampler, and Agilent 1100 UV-DAD-detector, and HP ChemStation software (Version A.10.03) on an IBM computer (operated by Windows XP professional), was used for this method. A Waters Symmetry ${ }^{\circledR} \mathrm{C} 18,3.5 \mu \mathrm{m}, 3.0 \mathrm{~mm} \times 150 \mathrm{~mm}$ analytical column was used with a Waters Symmetry ${ }^{\circledR} \mathrm{C} 18,5.0 \mu \mathrm{m}, 3.9 \mathrm{~mm} \times 20 \mathrm{~mm}$ guard column. An Eppendorf 5415D centrifuge (Hamberg, Germany) and Turbovap LV evaporator from Zymark (Hopkinton, MA, USA) were used in sample processing.

\subsection{Preparation of standards}

A clear stock solution of raltegravir was prepared at a concentration of $1 \mathrm{mg} / \mathrm{mL}$ using $5.46 \mathrm{mg}$ of raltegravir potassium dry base powder (molecular weight $482.51 \mathrm{~g}$ ) dissolved in $5.0 \mathrm{~mL}$ HPLC-grade water. The master stock solution was prepared by diluting $1.0 \mathrm{~mL}$ of the stock solution with $9.0 \mathrm{~mL}$ of HPLC-grade water to a final concentration of $100 \mu \mathrm{g} / \mathrm{mL}$. This 100 $\mu \mathrm{g} / \mathrm{mL}$ master stock solution was added to drug-free pooled human CVF diluted 1:3 with normal saline to give final working concentrations of $0.05,0.1,0.5,1.0,5.0$, and $10.0 \mu \mathrm{g} / \mathrm{mL}$. Quality control (QC) samples at a final concentration of $0.06,0.6$ and $6.0 \mu \mathrm{g} / \mathrm{mL}$ were also prepared using a 1:3 dilution of CVF. A 1:3 dilution of CVF was used to create the calibrators to ensure accurate pipetting of the viscous, mucous matrix, and homogenous dispersion of raltegravir within the matrix. To obtain drug-free pooled CVF, samples from healthy volunteers were obtained from the posterior fornix of the vagina via direct aspiration with a volumetric vaginal aspirator. The University of North Carolina (UNC) institutional review board approved the sample collection protocol and all subjects provided written informed consent before any collection procedures were performed. 


\subsection{Internal standard (IS) preparation}

Diazepam $(10 \mathrm{mg})$ was dissolved in a 50:50 solution of methanol and water to achieve a final concentration of $1 \mathrm{mg} / \mathrm{mL}$ (stock solution). From this solution, an aliquot was diluted in HPLCwater to a final concentration of $2 \mu \mathrm{g} / \mathrm{mL}$ (working solution).

\subsection{Simulated vaginal fluid (SVF) investigations}

Because of the scarcity of the CVF matrix, SVF based on the constituents and $\mathrm{pH}$ of human vaginal secretions [7], was tested to determine if it could reasonably substitute as the matrix for standard curves and QCs. If found to be accurate, precise, and comparable to actual CVF results with raltegravir, it could be used in place of donor drug-free CVF for calibration curves and QCs. The SVF consisted of the following ingredients: $1.76 \mathrm{~g}$ sodium chloride, $0.7 \mathrm{~g}$ potassium hydroxide, $0.111 \mathrm{~g}$ calcium hydroxide, $0.009 \mathrm{~g}$ bovine serum albumin, $1.0 \mathrm{~g}$ lactic acid, $0.5 \mathrm{~g}$ acetic acid, $0.08 \mathrm{~g}$ glycerol, $0.2 \mathrm{~g}$ urea, and $2.5 \mathrm{~g}$ glucose. This was brought to a volume of $500 \mathrm{~mL}$ with HPLC-grade water. The $\mathrm{pH}$ of the resulting solution was 4.2 .

\subsection{Sample collection and processing}

CVF samples were collected from healthy subjects that received an oral dose of raltegravir enrolled in a pharmacokinetic study. CVF was collected in the same manner as the drug-free pooled CVF used to make the calibrators. CVF was kept on ice before being stored in preweighed cryovials at $-80^{\circ} \mathrm{C}$ within $1 \mathrm{~h}$ of collection. Before processing, collection tubes were weighed to determine the weight of CVF collected (mg). To prepare for extraction, $100 \mu \mathrm{L}$ of calibrators made in a 1:3 dilution of CVF with normal saline, or $100 \mu \mathrm{L}$ of undiluted CVF sample collected in the pharmacokinetic study, were transferred to a $1.5 \mathrm{~mL}$ Eppendorf tube using a positive displacement pipettor. If $100 \mu \mathrm{L}$ of sample was not collected by the subject or could not be pipetted due to its viscous nature, a known amount that could be pipetted was used and the amount recorded. The peak area detected was adjusted for the volume pipetted compared to the $100 \mu \mathrm{L}$ standard. If it was not possible to accurately pipette a small volume of CVF, the perchloric acid and internal standard were added directly to the cryovial. The cryovial was vortexed so that the entire CVF sample was rinsed into the $1.5 \mathrm{~mL}$ Eppendorf tube. For these samples, the weight of CVF collected was used to estimate the volume of sample collected $(1 \mathrm{mg}=1 \mu \mathrm{L})$ in order to calculate the sample concentration.

To the $1.5 \mathrm{~mL}$ Eppendorf tubes, $200 \mu \mathrm{L}$ of $1 \%$ perchloric acid was added, along with $100 \mu \mathrm{L}$ of internal standard working solution (diazepam $2 \mu \mathrm{g} / \mathrm{mL}$ ). All tubes containing this mixture (CVF sample or calibrator $+1 \%$ perchloric acid + internal standard) were vortexed twice for $30 \mathrm{~s}$ each, and centrifuged at 13,000rpm for $5 \mathrm{~min}$. Three different concentrations of perchloric acid $(0 \%, 1 \%$, and $2.5 \%)$ were tested with QC samples to compare extraction efficiency.

\subsection{SPE method}

Solid-phase extraction Bond Elute ${ }^{\circledR}$ columns (1.0 mL, $100 \mathrm{mg}$, Varian, Harbor City, CA, USA) were placed in a vacuum elution manifold (20-SPE system, Waters, Milford, MA, USA). The cartridges were rinsed with $1.0 \mathrm{~mL}$ of methanol, and conditioned with $1.0 \mathrm{~mL}$ of $150 \mathrm{mM}$ ammonium acetate ( $\mathrm{pH}$ 5.0). The entire volume of supernatant after sample and calibrator processing was applied directly to the column. The sample mixtures were allowed to pass through the column bed with reduced pressure $(2-4 \mathrm{mmHg})$. The column was further washed with $1.0 \mathrm{~mL}$ of $5 \%$ methanol in $50 \mathrm{mM}$ ammonium acetate buffer (pH 6.7), and the bed was suctioned dry $(>5.0 \mathrm{mmHg}$ ) for at least $1 \mathrm{~min}$. The analyte and internal standard were then eluted with $500 \mu \mathrm{L}$ of $1.5 \%$ glacial acetic acid $(99.9 \%)$ in methanol into a clean $1.5 \mathrm{~mL}$ Eppendorf tube. The eluent was evaporated to dryness (approximately 50-60 min) under a gentle nitrogen stream at $45^{\circ} \mathrm{C}$, and the residue was reconstituted with $50 \mu \mathrm{L}$ of mobile phase (A). The resulting solution was carefully vortex-mixed twice for $30 \mathrm{~s}$ each, and centrifuged at 
$13,000 \mathrm{rpm}$ for $10 \mathrm{~min}$. The supernatant was transferred to $200 \mu \mathrm{L}$ HPLC microvials (Agilent Technologies) and $40 \mu \mathrm{L}$ injected (dead volume is less than $2 \mu \mathrm{L}$ ) onto the column.

\subsection{High-performance liquid chromatographic conditions}

Chromatographic separation was achieved using a Symmetry ${ }^{\circledR} \mathrm{C} 18$ analytical column (150 $\mathrm{mm} \times 3.0 \mathrm{~mm}, 3.5 \mu \mathrm{m}$ particle size, Waters Corp., Milford, MA, USA), with a Symmetry ${ }^{\circledR}$ C18 guard column $(20 \mathrm{~mm} \times 3.9 \mathrm{~mm}, 5 \mu \mathrm{m}$ particle size, Waters Corp.). The absorbance was monitored at $218 \mathrm{~nm}\left(\lambda_{\max }\right)$. Two mobile phase components were used. Mobile phase A consisted of $25 \mathrm{mMsodium}$ phosphate (monobasic) buffer, $20 \%$ acetonitrile, and $0.5 \mathrm{mM}$ TBAP (pH 4.0). Mobile phase B consisted of $19.5 \%$ acetonitrile, $80 \%$ methanol and $0.5 \%$ TFA. The analysis was performed using a binary linear gradient, transitioning from $70 \%$ to $3 \%$ (compared to $73 \%$ to $3 \%$ in the plasma method [6]) mobile phase A over the initial 11 min of run time, with 4 additional minutes of re-equilibration to initial conditions. A flow rate gradient was also employed, transitioning from $0.55 \mathrm{~mL} / \mathrm{min}$ to $0.60 \mathrm{~mL} / \mathrm{min}$ (compared to 0.55 to $0.70 \mathrm{~mL} / \mathrm{min}$ in the plasma method [6]) over the initial $10 \mathrm{~min}$, and returning to $0.55 \mathrm{~mL} / \mathrm{min}$ at $15 \mathrm{~min}$. The analysis was performed at $40{ }^{\circ} \mathrm{C}$.

\section{Results}

\subsection{Simulated vaginal fluid experiment}

The standard curve using SVF with raltegravir was linear and resulted in a regression coefficient $\left(r^{2}\right)$ of 0.995 . However, unacceptable variability in sample extraction was found with this matrix. The extraction efficiency ranged from $60 \%$ to $109 \%$ for the $0.06 \mathrm{mg} / \mathrm{L} \mathrm{QC}$ concentration, $66 \%$ to $93 \%$ for the $0.6 \mathrm{mg} / \mathrm{L} \mathrm{QC}$ concentration, and $82 \%$ to $94 \%$ for the 6.0 $\mathrm{mg} / \mathrm{L} \mathrm{QC}$ concentration over three separate days. Average extraction efficiency for these three concentrations over 3 days ranged from $70 \%$ to $99 \%$. Using this matrix, the range of $\%$ accuracies was $65-110 \%$ for the standard curve, and $80-127 \%$ for the QCs. Due to the inconsistent results obtained with SVF, development of the method continued with drug-free CVF obtained from healthy volunteers.

\subsection{Linearity, limit of quantification}

Because of the mucous, proteinacious nature of CVF, it was difficult to achieve a homogenous solution of drug and internal standard using undiluted matrix. After evaluating different dilutions of this matrix, it was determined that a 1:3 dilution of CVF to normal saline provided for the most accurate pipetting of sample, and this dilution was used to create calibrators for the method. Peak area RAL:IS ratios for the calibration standards were proportional to the concentration of raltegravir in plasma over the range tested. The calibration curves were fitted by linear weighted least-squares regression. The linear regression data for the calibration curves of the method consistently demonstrated a regression coefficient $\left(r^{2}\right) \geq 0.999$. The method was linear from 0.05 to $10.0 \mathrm{mg} / \mathrm{L}$. Linearity was also tested without the internal standard to determine the direct proportionality of RAL peak areas with their corresponding concentrations.

\subsection{Selectivity and specificity}

The approximate retention times for RAL and IS were 6.9 and $9.9 \mathrm{~min}$, respectively. There was minimal interference from endogenous substances with the analyte or internal standard in the CVF extracts. Fig. 1a and b illustrates a blank chromatogram of extracted CVF and the $0.06 \mathrm{mg} / \mathrm{L}$ QC sample, respectively. 


\subsection{Accuracy and precision}

Results of the method validation are shown in Table 1 . The average concentration calculated from the standard curve for all QCs at the $0.06,0.6$, and $6 \mathrm{mg} / \mathrm{L}$ levels were $.068,0.61$, and $6.54 \mathrm{mg} / \mathrm{L}$, respectively. All observed data (within-day and between-day accuracy [\% deviation] and precision [\% CV]) were below $15 \%$. The accuracy of RAL, represented as percent deviation from the nominal concentration, ranged from $1.2 \%$ to $11.0 \%$ for all three concentrations for both within-day and between-day analysis. The method's precision for RAL ranged from $1.1 \%$ to $12.6 \%$ for both within-day and between-day analysis.

\subsection{Extraction efficiency (recovery \%)}

The recovery of raltegravir after solid-phase extraction was determined by comparing the average observed peak area of extracted QCs in triplicate, to that of an expected peak area for each QC calculated from a solution of $10 \mu \mathrm{g} / \mathrm{mL}$ raltegravir in mobile phase A. Three sample preparation methods were compared to determine the amount of protein precipitation required: HPLC-grade $\mathrm{H}_{2} \mathrm{O}$ ( $0 \%$ perchloric acid), $1 \%$ perchloric acid, or $2.5 \%$ perchloric acid. Table 2 gives the percentage recovery for the three sample preparation techniques at the QC concentrations. When only water was added to the sample preparation, the $0.06 \mathrm{mg} / \mathrm{L}$ concentration did not have a detectable peak above baseline. In the higher concentrations, 0.6 and $6.0 \mathrm{mg} / \mathrm{L}$, it was evident in the chromatogram that there was endogenous interference present in the matrix. When added in a concentration of $2.5 \%$, the perchloric acid decreased the amount of endogenous interference. However, no peak was detected at $0.06 \mathrm{mg} / \mathrm{L}$, suggesting that the altered $\mathrm{pH}$ of the sample adversely affected the extraction of raltegravir in the solid-phase packing. Therefore, the concentration of perchloric acid was decreased to $1 \%$, and was found to have the best extraction efficiency with the least amount of interference.

\subsection{Method application}

This method was used to quantify raltegravir in $166 \mathrm{CVF}$ samples from 7 subjects enrolled in a pharmacokinetic study; the data for which has been recently presented [8]. The University of North Carolina (UNC) institutional review board approved all sample collections under study protocol and all subjects provided written informed consent before any study procedures were performed. Fig. 1c shows the analysis of one of these samples. Fig. 2 represents a mean (SE) pharmacokinetic concentration versus time profile of raltegravir in the CVF and blood plasma of 3 healthy volunteers. Subjects received raltegravir $400 \mathrm{mg}$ twice daily for 7 days. Samples were collected immediately following the last dose on day 7.

\section{Discussion}

This is the first method to quantify the novel integrase inhibitor raltegravir in cervicovaginal fluid. Only two methods have been published for measuring antiretroviral drug concentrations in CVF: pelvic lavage [4] and direct aspiration [5]. A lavage method can be semi-quantitative if a marker exists to estimate the amount of dilution. To our knowledge, no one has been able to identify a marker to accurately correct for the dilution factor in pelvic lavage. However, the direct aspiration method has been successfully used for quantifying nucleoside reverse transcriptase inhibitors, nonnucleoside reverse transcriptase inhibitors, protease inhibitors, and CCR5 receptor antagonists [9].

To pursue development of an assay for measuring raltegravir in directly aspirated CVF, we chose to modify our previously published method for measuring raltegravir in blood plasma [6]. In the plasma method, blood samples were collected from subjects in sodium EDTA tubes and centrifuged at $2800 \mathrm{rpm}$ for $15 \mathrm{~min}$ at $4{ }^{\circ} \mathrm{C}$. Plasma was transferred to clean cryovials and stored at $-70{ }^{\circ} \mathrm{C}$. Prior to extraction, all patient plasma samples were heated for $60 \mathrm{~min}$ at $58^{\circ}$ $\mathrm{C}$ to inactivate the HIV virus. Samples were brought to room temperature and applied directly 
to Bond Elute ${ }^{\circledR}$ columns for SPE. No further sample preparation was required prior to SPE. A binary linear mobile phase gradient of 73-3\% mobile phase A over the initial 11 min plus $4 \mathrm{~min}$ of re-equilibration to initial conditions was used. A flow rate gradient was also employed, transitioning from $0.55 \mathrm{~mL} / \mathrm{min}$ to $0.70 \mathrm{~mL} / \mathrm{min}$ over the initial $11 \mathrm{~min}$, and returning to 0.55 $\mathrm{mL} / \mathrm{min}$ at $15 \mathrm{~min}$. The method resulted in high extraction efficiency, accuracy, and precision.

However, successful implementation of this method for CVF required three major modifications. First, an additional sample preparation was used prior to application to the SPE columns. Pre-treatment with $1 \%$ perchloric acid followed by vortex and centrifugation was used to precipitate proteins in the biological matrix and therefore decrease the amount of endogenous interference. Notably, there was a limit to the amount of perchloric acid that could be added. If the $\mathrm{pH}$ dropped too low, the lower limit of detection was affected due to minimal drug extraction from the matrix. In addition, heating of CVF samples to inactivate HIV virus was not performed because these samples were collected from HIV-uninfected subjects.

Second, a factor adjustment of detected peak area was used in the CVF method when $100 \mu \mathrm{L}$ of CVF could not be pipetted with a positive displacement pipettor (either due to the viscosity of the matrix or because the subject was unable to collect $100 \mu \mathrm{L}$ ). For example, if only $40 \mu \mathrm{L}$ of CVF could be accurately pipetted, the resultant peak area was multiplied by 2.5 when comparing the results to the standard curve. For plasma, a positive displacement pipettor is unnecessary and a volume of $100 \mu \mathrm{L}$ is easily obtained. Therefore, no adjustment of peak area is necessary for plasma.

Third, the calibrators in the plasma method were made in undiluted plasma. However, a 1:3 dilution of CVF with normal saline was used to make these calibrators to allow homogenous dispersion of standards and accurate pipetting. In contrast, the CVF samples collected from subjects were not diluted or centrifuged. The CVF was pipetted directly from the cryovial to the Eppendorf tube (except in the cases where the CVF could not be accurately pipetted, in which the $1 \%$ perchloric acid and internal standard were added directly to the cryovial and the entire CVF sample was rinsed out). The entire mixture in the Eppendorf tube (undiluted CVF sample + perchloric acid + internal standard) was centrifuged, and that resulting supernatant was applied to the SPE column. For most samples, only $40 \mu \mathrm{L}$ of undiluted sample was pipetted into the $1.5 \mathrm{~mL}$ Eppendorf tube, resulting in a similar amount of matrix compared to the calibrator.

Minor modifications in the plasma method's [6] mobile phase binary linear and flow rate gradients did not adversely affect results. The retention times changed only by $0.2 \mathrm{~min}$ for raltegravir and $0.1 \mathrm{~min}$ for IS. The adjusted gradients were also used for plasma samples from the same pharmacokinetic study, and resulted in similar linearity, accuracy, and precision.

Although SVF was tested, unacceptable variability in the extraction of raltegravir precluded its use for calibrators and quality controls. SVF was designed to mimic vaginal $\mathrm{pH}$ and osmolarity [7], however there are several other components of vaginal fluid that are not accounted for in SVF. Vaginal fluid is composed of vaginal transudite, exudate from Bartholin's and Skenes's glands, exfoliated epithelial cells, residual urine, and fluids from the upper reproductive tract such as cervical mucus and endometrial and tubal fluids [7].

This method is sensitive (as evidenced by a LLD of $0.05 \mathrm{mg} / \mathrm{L}$ ) and specific (with minimal endogenous interference). The method also meets the FDA's recommendations for bioanalytical method validation [10] accuracy (\% deviation was within $\pm 20 \%$ of the theoretical value at the lower limit of quantification and within $\pm 15 \%$ of the theoretical value for other concentrations) and precision (\% CV for the lower limit of quantification did not exceed 20\%, and did not exceed $15 \%$ for all other concentrations). 
Because of the sensitive, specific, accurate, and precise aspects of this method, it has been used to analyze CVF samples from a clinical pharmacokinetic study [8]. The ability to detect antiretroviral drugs in female genital tract secretions is essential for understanding pharmacokinetic-pharmacodynamic investigations to inform clinical research in HIV prevention. This method is assisting in this effort.

\section{References}

1. Joint United Nations Programme on HIV/AIDS (UNAIDS). Report on the global HIV/AIDS epidemic, 2002. Geneva, Switzerland: World Health Organization; 2002 Jul.

2. Hosseinipour M, Cohen MS, Vernazza PL, Kashuba AD. Clin. Infect. Dis 2002;34:1391. [PubMed: 11981736]

3. Merck \& Company-Isentress Prescribing Information. 2009 Jan [accessed July 21, 2009]. p. 1Available at: http://www.merck.com/product/usa/pi_circulars/i/isentress/isentress_pi.pdf

4. Si-Mohamed A, Kazatchine M, Heard I, Goujon C, Prazuck T, Aymard G, Cessot G, Kuo YH, Bernard MC, Diquet B, Malkin JE, Gutmann L, Bélec L. J. Infect. Dis 2000;182:112. [PubMed: 10882588]

5. Dumond JB, Yeh RF, Patterson KB, Corbett AH, Jung BH, Rezk NL, Bridges AS, Stewart PW, Cohen MS, Kashuba AD. AIDS 2007;21:1899. [PubMed: 17721097]

6. Rezk NL, White N, Kashuba AD. Anal. Chim. Acta 2008;628:204. [PubMed: 18929009]

7. Owen DH, Katz DF. Contraception 1999;59:91. [PubMed: 10361623]

8. Jones, A.; Talameh, J.; Patterson, KB.; Rezk, NL.; Prince, H.; Kashuba, AD. Presented at the 10th International Workshop on Clinical Pharmacology of HIV Therapy; The Netherlands; April 15-17; Amsterdam. 2009.

9. Dumond JB, Patterson KB, Pecha AL, Werner RE, Andrews E, Damle B, Tressler R, Worsley J, Kashuba AD, Acquir J. Immune Defic. Syndr 2009;51:546.

10. U.S. Department of Health and Human Services Food and Drug Administration. Guidance for Industry: Bioanalytical Method Validation. 2001 May [accessed July 23, 2009]. Available at: http://www.fda.gov/downloads/Drugs/GuidanceComplianceRegulatoryInformation/Guidances/ UCM070107.pdf 
(a)

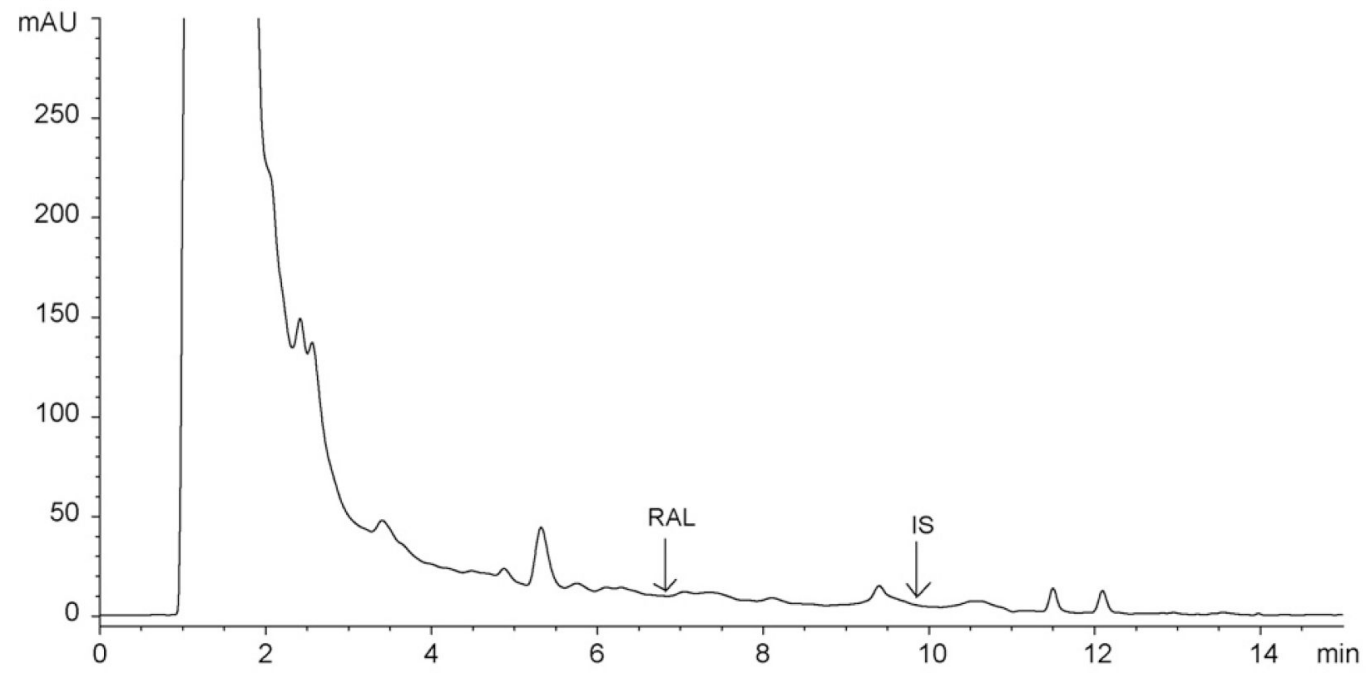

(b)

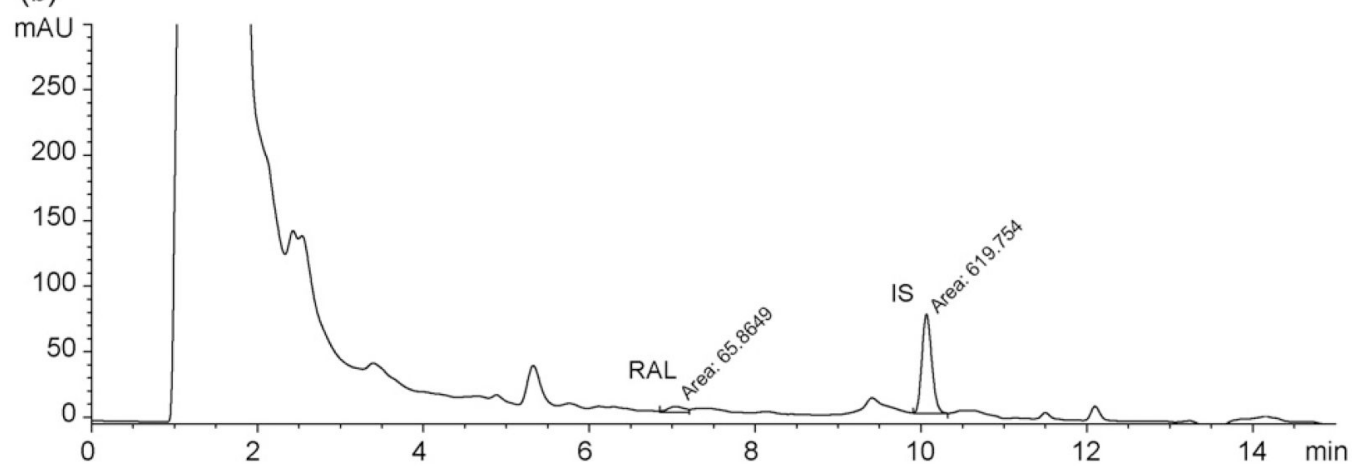

(c)

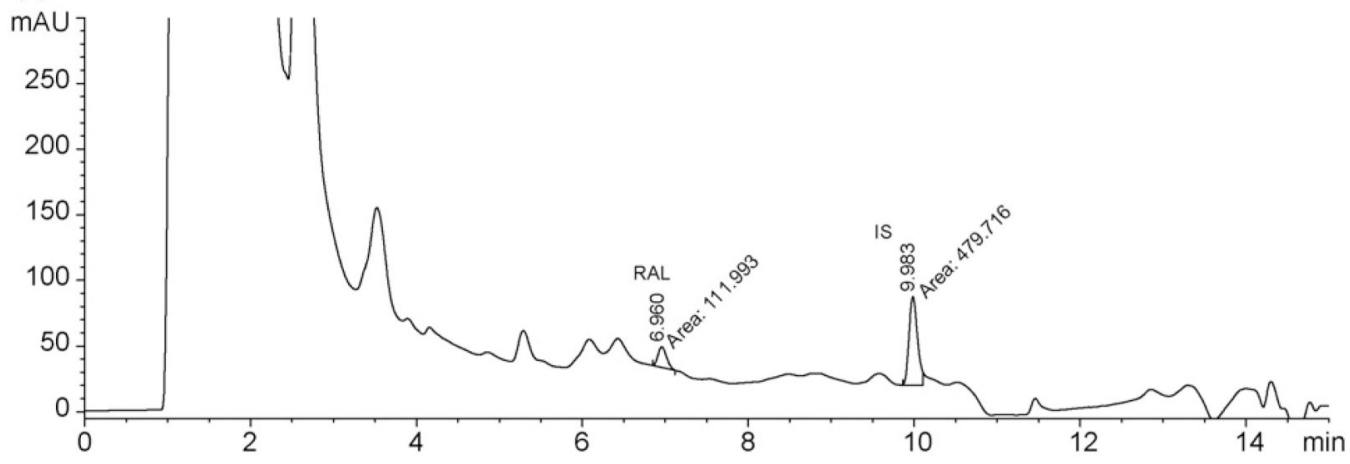

Fig. 1.

(a) Blank chromatogram of extracted CVF illustrating minimal endogenous interference with raltegravir (RAL) and internal standard (IS). (b) QC at $0.06 \mathrm{mg} / \mathrm{L}$ illustrating minimal endogenous interference with raltegravir (RAL) and internal standard (IS). (c) Chromatogram illustrating one CVF sample from pharmacokinetic study. 


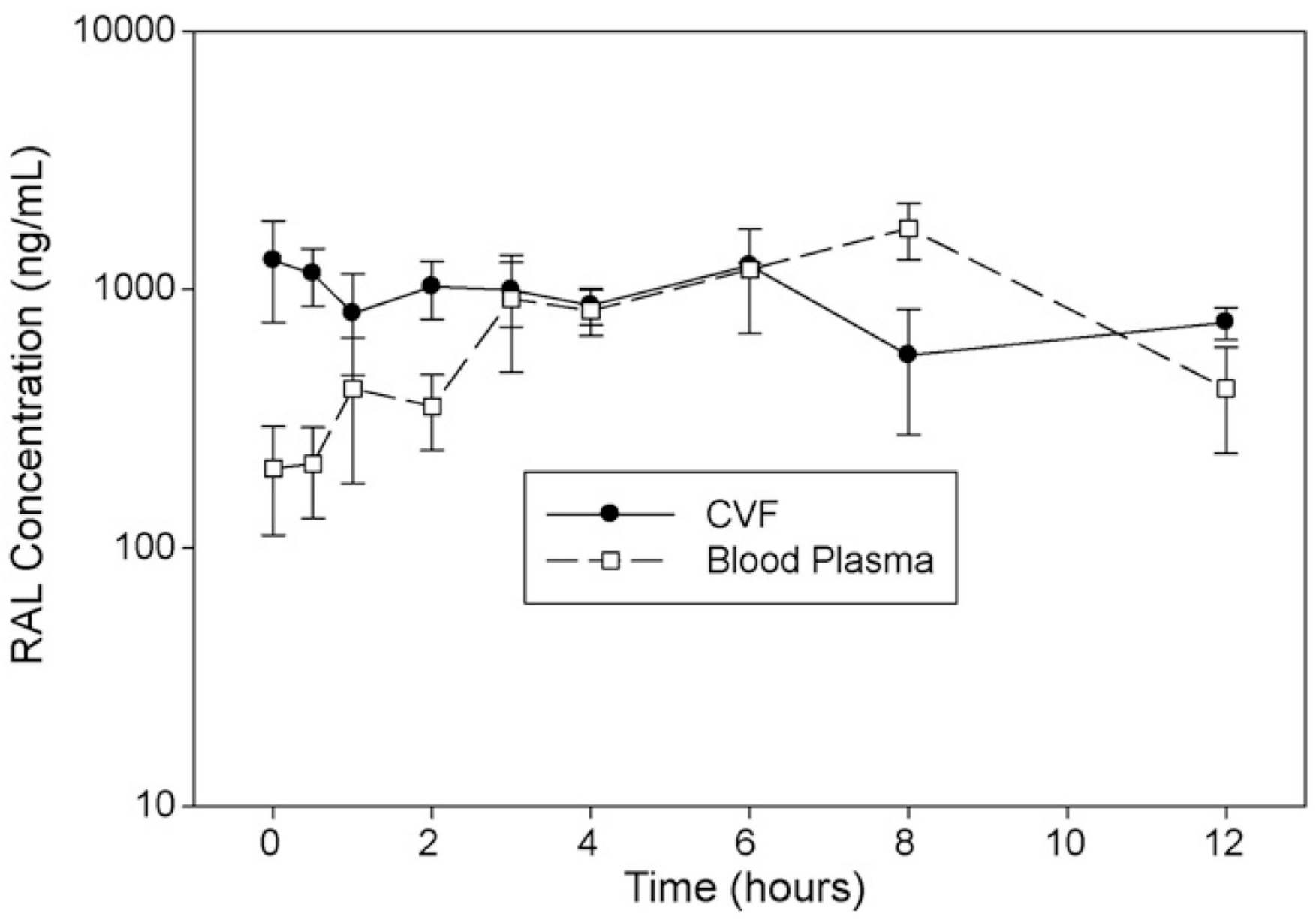

Fig. 2.

Mean (SE) concentration versus time pharmacokinetic profile in CVF and blood plasma on day 7 for three healthy subjects given raltegravir $400 \mathrm{mg}$ twice daily for 7 days. 


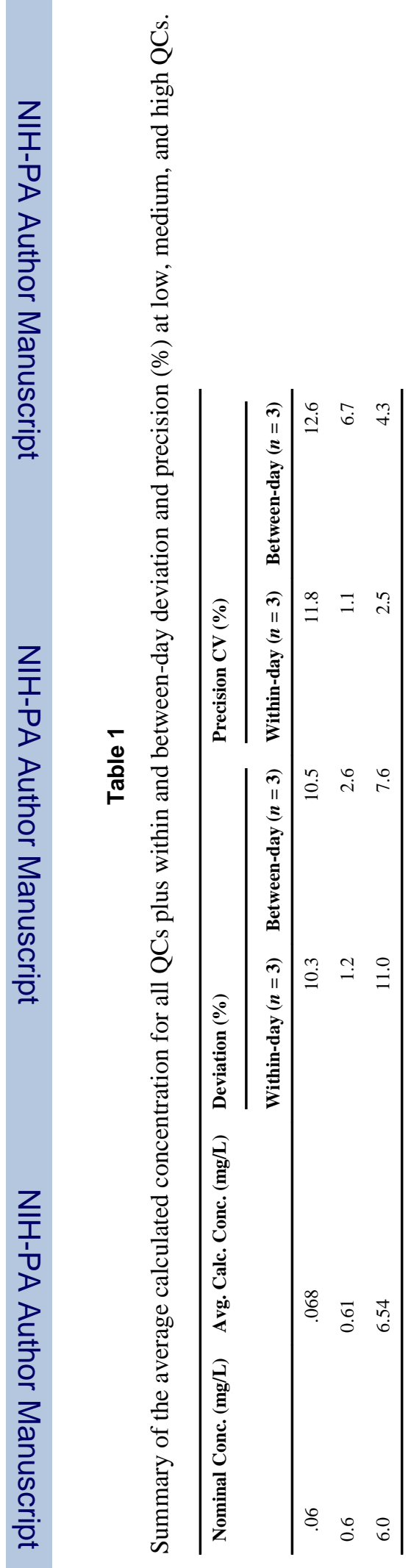




\section{Table 2}

Raltegravir extraction efficiency using various perchloric acid concentrations.

\begin{tabular}{llll}
\hline Concentration $(\mathbf{m g} / \mathbf{L})$ & $\mathbf{0 \%}$ perchloric acid $(\boldsymbol{n}=\mathbf{3})$ & $\mathbf{1 \%}$ perchloric acid $(\boldsymbol{n}=\mathbf{3})$ & $\mathbf{2 . 5 \%}$ perchloric acid $(\boldsymbol{n}=\mathbf{3})$ \\
\hline .06 & No peak & $59 \%$ & No peak \\
.60 & $80 \%$ & $58 \%$ & $58 \%$ \\
6.0 & $82 \%$ & $60 \%$ & $61 \%$ \\
\hline
\end{tabular}

